\title{
PREVALÊNCIA NA PERCEPÇÃO DA QUALIDADE DE VIDA, NAS DIMENSÕES DE SAÚDE FÍSICA/MENTAL NO AMBULATÓRIO DE DERMATOLOGIA DA UNIVERSIDADE CEUMA
}

\author{
PREVALENCE IN THE PERCEPTION OF THE QUALITY OF LIFE, IN THE \\ DIMENSIONS OF PHYSICAL / MENTAL HEALTH IN THE OUTPATIENT \\ DERMATOLOGY OF THE CEUMA UNIVERSITY
}

\author{
Rodrigo Sevinhago ${ }^{1}$; Matheus Cardoso Silva ${ }^{2}$; \\ Karine de Paiva Lima Nogueira Nunes ${ }^{3}$; Joana Kátya Veras Rodrigues Sampaio Nunes ${ }^{4}$
}

RESUMO: Esse estudo teve como objetivo avaliar o impacto das doenças crônicas não transmissíveis (DCNTs) e da qualidade de vida (QV), em pacientes que buscam atendimento médico na especialidade de Dermatologia, identificando a prevalência do nível da QV nos pacientes. As sensações físicas de dor e desconforto, não são as únicas variáveis elegíveis para a percepção da saúde, mas também envolvem consequências sociais e psicológicas da doença. Via de regra, as DCNTs, acometem pessoas de todas as faixas etárias e étnicas, desencadeando eventos de efeitos danosos, além de corroborarem para a coexistência de complicações a nível multidimensional na vida do paciente, gerando dano a sua QV. Trate-se de um estudo transversal, bibliográfico, descritivo e sistematizado com pacientes de ambos os sexos, que frequentaram o ambulatório de dermatologia na Universidade Ceuma, no período de maio 2016 a dezembro 2016. Sendo os dados obtidos a partir de entrevistas individualizadas na pré ou pós consulta. Foi utilizado o SF-36 na avaliação da QV, com o objetivo de avaliar a prevalência da pior percepção da QV. Dos 38 pacientes avaliados, $7(18,4 \%)$ apresentavam pior escore da $\mathrm{QV}$, desses $71,4 \%$ eram mulheres, $100 \%$ pior escore no aspecto emocional, $85,7 \%$ pior escore no aspecto físico e $71,4 \%$ pior escore na saúde mental. A partir dos resultados pode concluir que é significativa a prevalência da pior percepção da QV nos pacientes que frequentam o ambulatório e que medidas que busquem melhorias do aspecto emocional, físico e de saúde mental devem ser instituídas para efetividade do tratamento.

PALAVRAS-CHAVE: Doenças crônicas não transmissíveis; Qualidade de Vida; SF-36.

1 - Acadêmico do Curso de Medicina, $7^{\circ}$ período, UNICEUMA. Vinculado ao Núcleo de Pesquisa Multiprofissional em Saúde/MULTIMED/CEUMA/CNPQ. E-mail: rodrigo.sevi@gmail.com

2 - Acadêmico do Curso de Medicina, $7^{\circ}$ período, UNICEUMA. Vinculado ao Núcleo de Pesquisa Multiprofissional em Saúde/MULTIMED/CEUMA/CNPQ. E-mail: Matheus-cs@outlook.com

3 - Mestra em Gestão de Programas de Serviços de Saúde pela UNICEUMA, Docente titular da UNICEUMA. Pesquisadora do Núcleo de Pesquisa Multiprofissional em Saúde/MULTIMED/CEUMA/CNPQ e Pesquisadora do Grupo de Estudo e Pesquisa em Gestalt-Terapia/GEPEGEST/CEUMA/CNPQ. E-mail: karine_nunes@terra.com.br 4 - Mestra em Ciências do Programa de Pós Graduação em Ciências da Saúde - UFMA. Doutoranda em Ciências da Saúde - UFMA. Docente do curso de psicologia e medicina da UNICEUMA. Pesquisadora do Núcleo de Pesquisa Multiprofissional em Saúde/MULTIMED/CEUMA/CNPQ e Núcleo de Pesquisa em Saúde Coletiva do Maranhão/NUPESCMA/CNPQ. E-mail: iap_psy@ig.com.br 


\begin{abstract}
The purpose of this study was to evaluate the impact of chronic noncommunicable diseases (NCDs) and quality of life (QoL) on patients seeking medical attention in the specialty of Dermatology, identifying the prevalence of QoL in patients. Physical sensations of pain and discomfort are not the only variables eligible for health perception, but also involve social and psychological consequences of the disease. As a rule, CNCDs affect people of all ages and ethnic groups, triggering events of harmful effects, and corroborate the coexistence of complications at the multidimensional level in the patient's life, causing damage to his QOL. This is a cross-sectional, bibliographic, descriptive and systematized study with patients of both sexes, who attended the dermatology outpatient clinic at Ceuma University, from May 2016 to December 2016. The data were obtained from individualized interviews in the pre- or after consultation. SF-36 was used in the evaluation of QoL, with the objective of evaluating the prevalence of worse perception of QoL. Of the 38 patients evaluated, 7 (18.4\%) had a worse QL score, $71.4 \%$ were women, $100 \%$ had a worse emotional score, $85.6 \%$ had a worse physical score, and $71.4 \%$ had a worse score mental health. From the results, it can be concluded that the prevalence of the worse perception of QoL is significant in patients attending the outpatient clinic and that measures that seek improvements in the emotional, physical and mental health aspects should be instituted for treatment effectiveness.
\end{abstract}

KEYWORDS: Chronic noncommunicable diseases; Quality of life; SF-36. 


\section{Introdução}

Documentos da Organização Mundial da Saúde (OMS) reconhecem que é direito de todo ser humano, possuir e viver uma vida saudável (WHO, 2001). Mas, pesquisas em todo o mundo apontam que, existem vários fatores de riscos que podem expor a qualidade de vida de uma pessoa, e via de regra esses fatores estão associados a doenças crônicas não transmissíveis (DCNT's) (WESONGA et al., 2015; WESONGA-b et al., 2016).

As DCNT's, são classificadas como agravos que apresentam geralmente características como: serem permanentes, levarem a incapacidade/deficiência residual, serem causadas por alterações patológicas irreversíveis, exigirem formação especial do doente para a reabilitação, ou podem exigir longos períodos de supervisão, observação ou cuidados (ABEGUNDE, 2007).

No contexto epidemiológico as DCNT's, apresentam elevadas taxas de morbidade e mortalidade, ao passo que são responsáveis por cerca de $70 \%$ das mortes em todo o mundo, estudos contrastam que $75 \%$ dessas mortes ocorrem em países não desenvolvidos ou subdesenvolvidos (CUNDALE, 2017). Mundialmente, elas compreendem: o diabetes, as doenças cardiovasculares, o câncer e as doenças respiratórias crônicas (CUNDALE, 2017).

Muitas doenças pertencentes a esse conjunto apresentam fatores de risco comuns como a hipertensão, obesidade, depressão e ansiedade além de demandarem assistência continuada de serviços e ônus progressivo, proporcional ao envelhecimento de cada indivíduo e também da população provocando ainda mudanças na rotina e no planejamento das atividades, especialmente nos casos de doenças incapacitantes, em que o doente deixa de exercer suas atividades cotidianas (ACHUTTI et al., 2004; SMELTZER; BARE; BRUNNER \& SUDDARTH, 2005).

Identificar os fatores de risco além de implementar medidas preventivas podem reduzir de maneira efetiva a manifestação da doença além da morte por DCNT's (KOŁTUNIUK; ROSIŃCZUK, 2018). Entretanto, é notória a diversidade dos fatores de risco modificáveis apresentados por cada pessoa além do fator genético atenuante, que sinaliza para o aumento acumulado dos fatores de risco predisponentes (CUREAU et al., 2014; WESONGA et al., 2016). 


\section{Material e Métodos}

Trata-se de um estudo transversal, bibliográfico, descritivo e sistematizado com pacientes de ambos os sexos, que frequentaram o ambulatório da clínica de Dermatologia da Universidade CEUMA, no período de Maio de 2016 a Dezembro de 2016, aprovado pelo Comitê de Ética em Pesquisa da instituição ( $\mathrm{N}^{\circ}$. do parecer consubstanciado: 1.199.341).

Tendo como critério de não inclusão, não ter diagnóstico médico de DCNT's, doença crônica que limitasse sua participação ou expectativa de vida, possuir outros transtornos psiquiátricos que pudessem impedir a compreensão e comunicação durante a entrevista e os que se recusaram a assinar o Termo de Consentimento Livre e Esclarecido (TCLE) e como critério de exclusão aqueles que por algum motivo abandonaram o estudo em qualquer momento.

Os instrumentos de coleta de dados foram os questionários sociodemográficos (idade, cor da pele autodeclarada, situação conjugal, ocupação, renda familiar e escolaridade), antecedentes pessoais e familiares patológicos, hábitos de vida (tabagismo, alcoolismo e atividade física), comorbidades não transmissíveis e o de avaliação da qualidade de vida, formulário SF-36.

O formulário validado, SF-36 é um instrumento multidimensional que apresenta oito domínios para avaliação da qualidade de vida, sendo divididos em dois grandes componentes, o físico (capacidade funcional, aspecto físico, dor, estado geral de saúde) e mental (vitalidade, aspecto social, aspecto emocional, saúde mental). (SILVA; FERREIRA, 2011). Dessa forma, é gerado um escore próprio para cada questão e posteriormente são convertidos para uma escala de 0 a 100, em que valores próximos a zero inferem a pior percepção da qualidade de vida, e escores numéricos próximos a 100 revelam boa percepção da qualidade de vida e consequentemente da saúde (SILVA; FERREIRA, 2011).

Os dados foram obtidos a partir de entrevistas individualizadas realizadas na pré ou pós consulta, dos pacientes que buscaram atendimento na clínica de dermatologia.

\section{Resultados e discussão}

Dentre os 38 pacientes avaliados, notou-se uma frequência de 73,7\% (28) de mulheres, mais concentrados na faixa etária de 16 a 25 anos, 31,0\% (12), a raça parda foi a mais prevalente com $44,7 \%$ (17). Entretanto, considerando os pacientes que obtiveram a pior percepção da 
qualidade de vida, notou-se uma frequência de 20,0\% (2) de homens, mais concentrados na faixa etária de 26 a 35 anos, 31,0\% (3), a raça parda foi a mais prevalente com 57,1\% (4) (Tabela 1).

Tabela 1. Distribuição das variáveis sóciodemográficas (sexo, idade e raça) dos pacientes que frequentam o ambulatório de Dermatologia da Universidade Ceuma, em relação a melhor e pior percepção da QV. Sevinhago, R. São Luís, 2018.

\begin{tabular}{|c|c|c|c|c|c|c|}
\hline \multirow{3}{*}{ Variáveis } & \multirow{2}{*}{\multicolumn{2}{|c|}{ Total }} & \multicolumn{4}{|c|}{ Pacientes que responderam o SF-36 } \\
\hline & & & \multicolumn{2}{|c|}{ Melhor Percepção } & \multicolumn{2}{|c|}{ Pior Percepção } \\
\hline & $\mathbf{N}$ & $\%$ & $\mathbf{N}$ & $\%$ & $\mathbf{N}$ & $\%$ \\
\hline \multicolumn{7}{|l|}{ Sexo } \\
\hline Masculino & 10 & 26,3 & 8 & 80,0 & 2 & 20,0 \\
\hline Feminino & 28 & 73,7 & 23 & 82,1 & 5 & 17,9 \\
\hline \multicolumn{7}{|l|}{ Idade (anos) } \\
\hline 16 a 25 & 16 & 42,1 & 16 & 100,0 & 0 & 0 \\
\hline 26 a 35 & 6 & 15,9 & 3 & 50,0 & 3 & 50,0 \\
\hline 36 a 45 & 4 & 10,5 & 3 & 75,0 & 1 & 26,0 \\
\hline 46 a 55 & 7 & 18,4 & 4 & 57,1 & 3 & 42,9 \\
\hline 56 a 65 & 4 & 10,5 & 4 & 100,0 & 0 & 0 \\
\hline 66 ou mais & 1 & 2,6 & 1 & 100,0 & 0 & 0 \\
\hline \multicolumn{7}{|l|}{ Raça } \\
\hline Branca & 11 & 26,3 & 11 & 100,0 & 0 & 0 \\
\hline Parda & 17 & 44,7 & 13 & 42,9 & 4 & 57,1 \\
\hline Negra & 10 & 26,3 & 7 & 57,1 & 3 & 42,9 \\
\hline TOTAL & 38 & 100,0 & 31 & 81,6 & 7 & 18,4 \\
\hline
\end{tabular}

Já em termos de frequência relativa, dos 38 pacientes avaliados, $7(18,4 \%)$ apresentavam pior escore da QV (Tabela 1), desses 71,4\% (5) eram mulheres, 100\% (7) pior escore no aspecto emocional, 85,7\% (6) pior escore no aspecto físico e 71,4\% (5) pior escore na saúde mental (Gráfico 1).

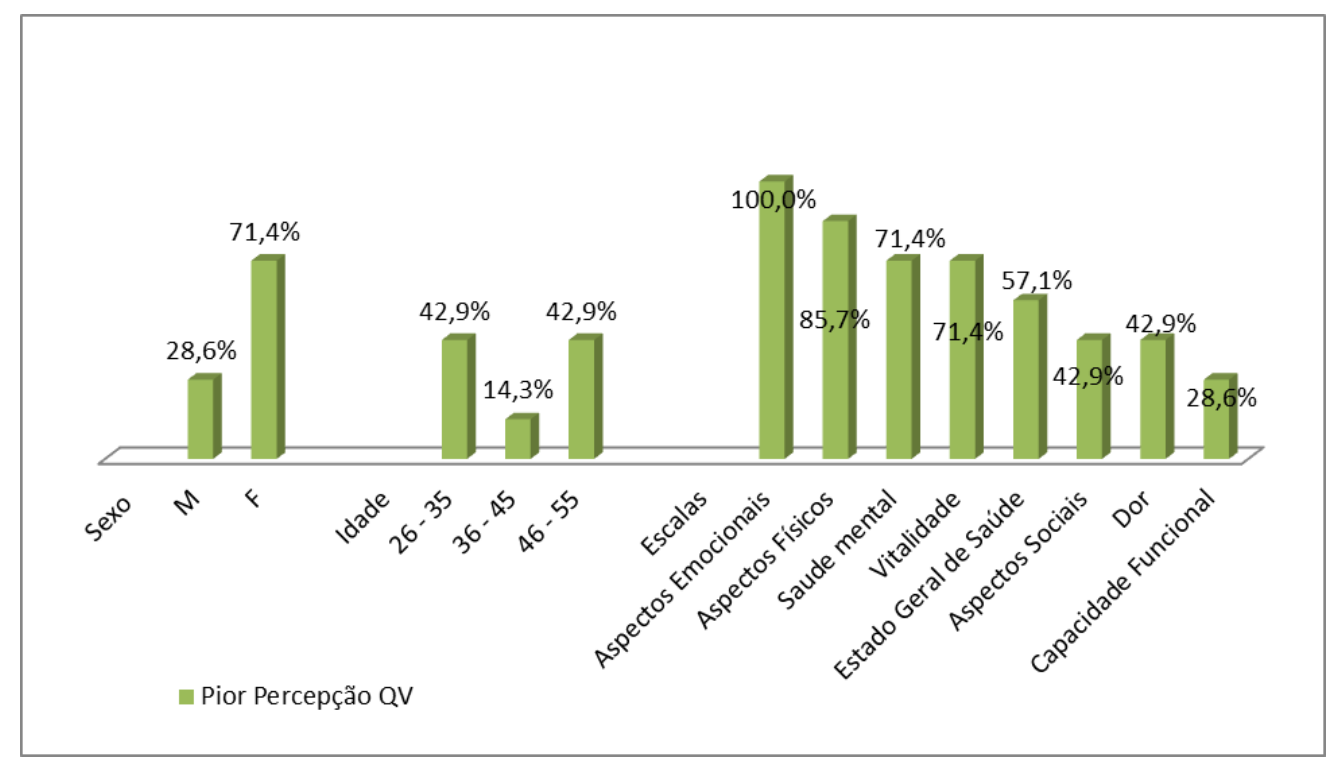


Gráfico 1 - Frequência Relativa das variáveis sexo, idade e escalas do SF-36 em função da pior percepção da QV avaliada no estudo. Sevinhago, R. São Luís, 2018.

Considerando que entre os pacientes que apresentaram pontuação menor que 50 em qualquer um dos domínios, notou-se que os domínios Aspectos Emocionais e Vitalidade foram os mais prevalentes com 17,2\% (10) cada, seguidos pelo domínio Aspectos Físicos com 15,5\% (9), domínio Saúde Mental com 13,9\% (8) (Tabela 2).

Ainda pela Tabela 2, é possível inferir que a pior percepção prevalece ou iguala entre os aos pacientes que apresentaram pontuação menor que 50 em qualquer um dos domínios, exceto no domínio da Dor, pois o mesmo se trata de um domínio mais subjetivo a percepção da QV e bem estar físico.

Tabela 2. Escalas avaliadas pelo SF-36 dos pacientes que frequentam o ambulatório de Dermatologia da Universidade Ceuma, em relação a melhor e pior percepção da QV nos pacientes que apresentaram pontuação menor que 50 em qualquer dos domínios. Sevinhago, R. São Luís,

2018.

\begin{tabular}{lcccccc}
\hline \multirow{2}{*}{ Variáveis } & \multicolumn{2}{c}{ Total } & \multicolumn{3}{c}{ Pacientes que responderam o SF-36 } \\
\cline { 4 - 7 } & & & Melhor Percepção & \multicolumn{2}{c}{ Pior Percepção } \\
\cline { 2 - 7 } \multicolumn{1}{c}{ Domínios } & $\mathbf{N}$ & $\mathbf{\%}$ & $\mathbf{N}$ & $\mathbf{\%}$ & $\mathbf{N}$ & $\mathbf{\%}$ \\
\hline Aspectos Emocionais & 10 & 17,2 & 3 & 30,0 & 7 & 70,0 \\
Vitalidade & 10 & 17,2 & 5 & 50,0 & 5 & 50,0 \\
Aspectos Físicos & 9 & 15,5 & 3 & 33,3 & 6 & 66,7 \\
Saúde mental & 8 & 13,9 & 3 & 37,5 & 5 & 62,5 \\
Estado Geral de Saúde & 7 & 12,1 & 3 & 42,9 & 4 & 57,1 \\
Dor & 7 & 12,1 & 4 & 57,1 & 3 & 42,9 \\
Aspectos Sociais & 5 & 8,6 & 2 & 40,0 & 3 & 60,0 \\
Capacidade Funcional & 2 & 3,4 & 0 & 0 & 2 & 100,0 \\
\hline \multicolumn{1}{c}{ TOTAL } & $\mathbf{5 8}$ & $\mathbf{1 0 0 , 0}$ & $\mathbf{2 3}$ & $\mathbf{3 9 , 7}$ & $\mathbf{3 5}$ & $\mathbf{6 0 , 3}$ \\
\hline
\end{tabular}

\section{Conclusões}

A partir dos resultados, é possível inferir que é significativa a prevalência da pior percepção da QV nos pacientes que frequentam o ambulatório de dermatologia e que instituir medidas que busquem melhorias do aspecto emocional, físico e de saúde mental deve ser realizado para efetividade do tratamento, minimizando assim a atenuação dos fatores de risco, pois a emoção influência diretamente o sistema de cura natural do corpo.

Ainda os dados aqui apresentados contribuíram de maneira ímpar para o desenvolvimento científico, visto que o plano de ações estratégicas para o enfrentamento das DCNT's no Brasil, 
Revista Ceuma Perspectivas, Edição Especial V Congresso de Saúde e Bem Estar Ceuma. Vol. 30, nº2, 2017.

ISSN Eletrônico: 2525-5576.

ainda encontra-se em vigor, dessa maneira esse trabalho prosperará com resultados na área ainda poupo difundida, servindo de dados para ações governamentais e também estatísticas.

\section{Referências}

ABEGUNDE, D.O.; e et al. The burden and costs of chronic diseases in low-income and middleincome countries. The Lancet, London: The Lancet Publications; Oxford: Elsevier, v. 370, n. 9603, p. 1929-1938, Dec. 8, 2007. Disponível em:

<http://www.who.int/choice/publications/p_2007_Chronic_disease_burden_Lancet.pdf >. Acesso em: 21 mar. 2018.

ACHUTTI, A.; AZAMBUJA, M.I.R. Chronic non-communicable diseases in Brazil: the health care system and the social security sector. Ciência \& Saúde Coletiva, 9(4):833-840, 2004.

CUNDALE, K.; WROE, E.; MATANJE-MWAGOMBA, B.L., MUULA, A.S.; GUPTA, N.; BERMAN, J.; e et al. Reframing noncommunicable diseases and injuries for the poorest Malawians: the Malawi National NCDI Poverty Commission. Malawi Medical Journal, 29(2), 194-197, 2017. Disponível em: <https://www.ncbi.nlm.nih.gov/pmc/articles/PMC5610295/>. Acesso: 20 mar. 2018.

CUREAU, F.V.; DUARTE, P.; SANTOS, D.L.; e et al. Clustering of risk factors for noncommunicable diseases in Brazilian adolescents: Prevalence and correlates. J Phys Act Health. 2014;11. Disponível em: <https://www.ncbi.nlm.nih.gov/pubmed/23676377>. 21 mar. 2018.

KOŁTUNIUK, A; ROSIŃCZUK, J. The influence of gender on selected risk factors for chronic non-communicable diseases in patients hospitalized in surgical wards: A cross-sectional study. Adv Clin Exp Med. 2018. Dispoonível em: <https://www.ncbi.nlm.nih.gov/pubmed/2955803>. Acesso em: 22 mar. 2018.

SILVA, C.A.S.; FERREIRA, C.E.S. Efeitos de um programa de reabilitação cardíaca na qualidade de vida relacionada à saúde (SF-36). Educação Física em Revista [on-line]. 2011;5(1). Disponível em: <http://www.onlineijcs.org/english/sumario/26/26-5/artigo2.asp>.

SMELTZER, S.C.; BARE, B.G.; BRUNNER \& SUDDARTH. Tratado de enfermagem médicocirúrgica. 10a ed. Rio de Janeiro: Guanabara Koogan; 2005. p.275-80. Karine Zortéa Departamento de Psiquiatria, Hospital de Clínicas de Porto Alegre, Universidade Federal do Rio Grande do Sul, Porto Alegre, RS - Brasil: Quality of Life in Chronic Diseases. 
WESONGA R.; GUWATUDDE, D.; BAHENDEKA, S.K.; e et al. Burden of cumulative risk factors associated with non-communicable diseases among adults in Uganda: evidence from a national baseline survey. International Journal for Equity in Health. 2016; Disponível em: <https://www.ncbi.nlm.nih.gov/pmc/articles/PMC5133748/>. Acesso em: 21 mar. 2018.

WESONGA, R.; OWINO, A.; SSEKIBOOBO, A.; e et al. Health and human rights: a statistical measurement framework using household survey data in Uganda. BMC Int Health Hum Rights. 2015;15. Disponível em:

<https://www.ncbi.nlm.nih.gov/pmc/articles/PMC4429314/?report=reader>. Acesso em: 21 mar. 2018.

WHO - World Health Organization. STEPS instruments for NCD risk factors (core and expanded version 1.4): the WHO STEPwise approach to Surveillance of noncommunicable diseases (STEPS) 2001). 